\title{
Road to CESR (Article 14)- Is it for me?
}

\author{
Chetan M. Pataki ${ }^{1}$
}

\begin{abstract}
"What lies behind us $\mathfrak{b}$ what lies ahead of us are tiny matters to what lies within us!" -Ralph Waldo Emerson
\end{abstract}

Many clinicians across the world aspire to come to United Kingdom and work in NHS. Initial months could be challenging to work in entirely new healthcare system. With some help from good colleagues, transition for overseas doctors can be less overwhelming.

As an International Medical Graduate (IMG), there are several options for career progression in UK. Many doctors enter the UK formal training program, however, Certificate of Eligibility for Specialist Registration (CESR) is an alternative pathway to achieve CCT. The CESR route could be helpful for clinicians trained in countries outside the UK or European Union as their training from their country of origin might not be recognized equivalent to CCT by General Medical Council (GMC). After obtaining CESR certification, your name is added to the specialist register which is an absolute necessity to obtain a substantive consultant position in the UK.

There is an ever-increasing requirement for doctors in the UK due to staff shortage and now Covid-19 pandemic has pushed many trusts against the wall to fill these positions. Apart from recruiting overseas doctors, CESR certification has proven to be helpful in filling up these positions for the trusts. The GMC made some favourable changes in the CESR process in recent years which has encouraged many career grade/staff grade doctors to progress. On other hand, trainees who fail to obtain training post after several attempts are opting CESR as an alternative route to progress. Annual statistics published by the GMC indicate that there is rising trend in number of applications for CESR certification in all specialties. There is a gradual change in perception of CESR as an equally attractive route for career progression.

However, there are many unknowns in this pathway as this has been a path less travelled. There is a general perception that CESR certification is difficult to achieve. I intend to help those aspirants who wish to take this path. We will be discussing some of the intricacies of this pathway.
As I mentioned earlier, there are two routes to progress in any specialty in the UK to Consultant level.

\section{Progression through training (CCT/CCT-CP)}

This is the formal training route of progression where one has to apply and enter a training post (subject to eligibility) in the UK. Once successfully enrolled in a training post, it is very well laid out path to follow. Duration of training can vary as per specialty ranging from 3 to 7 years. Some trainees can seek exemption in training period depending on work experience after consideration by Royal Colleges on a case-by-case basis. There is provision for less than full time (LTFT) training, however, this will increase duration of the training.

In my opinion, it will be helpful to go down this route for those who never had formal training or who left training before completion. This could also be a convenient option for some doctors who are happy to get trained again despite been trained in past. It is an entirely different process to prepare and apply for specialty training and it is beyond scope of this article.

\section{Certificate of Eligibility for Specialist Registration (CESR/Article 14)}

CESR CCT was formerly known as Article 14 and the GMC and respective Royal colleges have provided specialty specific guidance for this pathway. As this is not part of a UK training program, one has to pave their own path to obtain documentation of equivalence similar to the standard of a UK trainee. The amount of documentation could range up to 1200 pages as advised by GMC. Dedication, devotion and determination (3D) lie at the heart of this process to gather such detailed specific documentation of competence in clinical and non-clinical domains. It might take a variable period of time to complete this paperwork and it is a humongous task to achieve. We will explore this option in detail in this article.

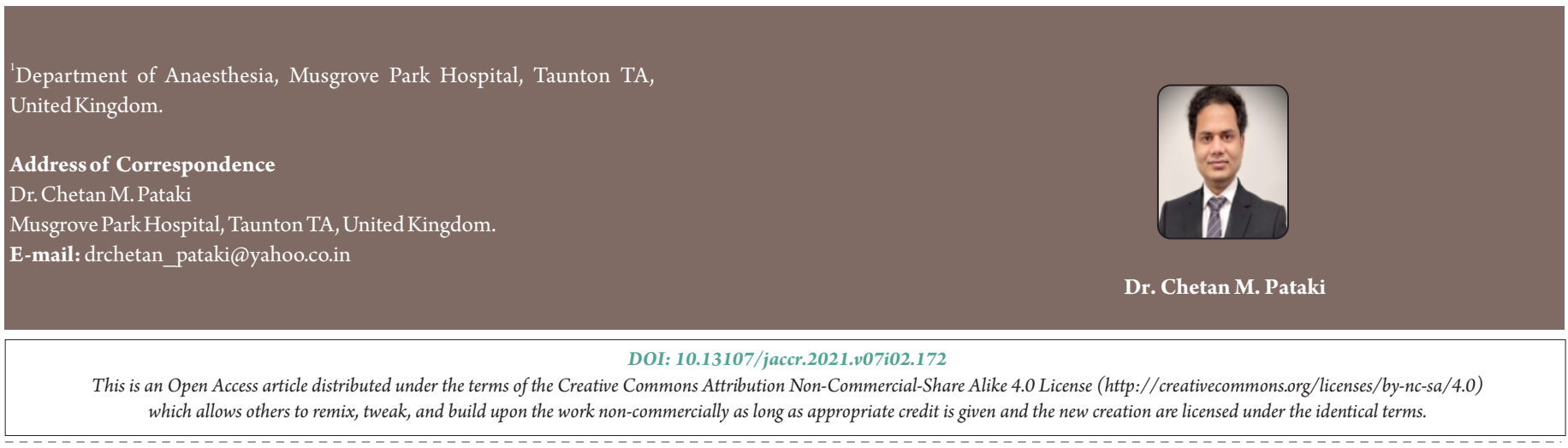


In my opinion, the few factors could be helpful to make an informed decision if one should go into training or down the CESR route. These are-

- Any previous training in your specialty: If you have adequate knowledge, skills as well as completed your training (outside UK) in past, then filling up gaps in knowledge (if any) or training could be easier. On the other hand, if someone has inadequate or no training in past experience, CESR could be much more challenging as there will be a large void to fill in. The benefit of this route is that you can progress at your own pace if that is what you prefer. It could be helpful to maintain good personal and professional life balance and avoid hassle of frequently switching workplaces like trainees.

- Personal factors: Whether you choose formal training or CESR pathway, it will take a significant amount of time and energy to pursue the cause.

In my opinion, the CESR route could be even more challenging in terms of arranging training and paperwork compared to a formal training program.

It is challenging to get paperwork sorted on many fronts. We will discuss this in further detail as this is the most crucial part of process.

- Paperwork from past: Any amount of appropriate evidence from past about training or work, if incorporated in your CESR application, will minimize the duration required to collect evidence prospectively.

On the other hand, if you don't have paperwork/evidence from past, even if you have vast experience, it is as good as non-existent unless proven otherwise. Many doctors do not have sufficient evidence in clinical or non-clinical components due to differences in healthcare systems. If you gather any evidence retrospectively, the GMC gives less weight to this paperwork. All the paperwork gathered needs to be submitted to the GMC. The GMC will be advised by the Royal College of that particular specialty in form of recommendations. When both these bodies are satisfied with the quality and quantity of evidence, application is accepted for entry onto the Specialist register. All the evidence provided in application should be verified from a hospital as the GMC will contact those verifiers for authenticity.

- Paperwork for Clinical domains: Identifying the lacunae in previous clinical experience or evidence by comparing it with UK Training Curriculum is pivotal at beginning. You can work towards bridging the gap by providing evidence for successful application. Work towards providing further UK experience if you have past experience/paperwork.

The Royal College and GMC website provides specialty specific guidance to map competencies and is useful to make a successful strategy. Keep checking the recent curriculum from college websites as it gets updated frequently.

In my opinion, you should choose a place of work in the UK carefully. If you have support from a trust to obtain CESR paperwork, you can collect paperwork meticulously in good time.
- Paperwork for Non-clinical domains: This is another crucial piece of evidence which is equally important for successful CESR application. Non-clinical skills like Team working, Leadership, Management. Education, Innovation, Academic and Research activities are given equal importance during scrutiny of your application by the GMC and Royal colleges. Engaging in such activities to gather evidence is very important.

In my opinion, if you are in non-training post in UK, seek help to work on projects like Quality improvement, Audits, research project, teaching program at local/regional/national level. There are many courses to support your past or ongoing activities like Leadership and management or Train the trainer but courses would not suffice as stand-alone evidence for these domains.

- Arranging paperwork: After gathering all the paperwork, it is a crucial step to submit the paperwork in the most presentable way. The GMC is very particular about organization of evidence and have provided many resources on their website. The GMC accepts only online application for CESR with minimal hard copy evidence like degrees and specialization certificates obtained outside UK and it should be authenticated by a solicitor. If you have access to an electronic portfolio, it will automatically arrange evidence as you upload it. I found this very helpful to keep the majority of my paperwork and logbooks in one place. For any non-UK work evidence, you have to find a convenient way to organize it to make it presentable as per recommendation by the GMC.

- Duration: Duration to gather such a detailed paperwork is variable based on previous experience. All the evidence should be within 5 years from date of submission. Minimum of 7 years of clinical experience is expected in specialty you are planning to apply for the CESR. The time required to collect this paperwork could be 2 years at best while in some cases it might take even longer than 5 years. Individual perseverance as well as help from your department could be a deciding. A helpful department with enthusiastic colleagues could shorten this duration.

In my opinion, having a mentor, supervisor or buddy might be beneficial to guide and help you.

- Application from outside the UK: Some clinicians decide to apply for the CESR from their home country outside the UK. You can certainly apply for CESR from outside the UK but this cannot be generalized to all specialties. You need to find appropriate guidance from the respective Royal College website or GMC website. To be eligible for CESR, many specialties require a training post for at least 6 months period in UK or abroad. MTI fellow post is also considered as a training post.

In my opinion, application from outside the UK could be challenging, however, this cannot be generalized.

\section{Useful tips:}

- Read the guidance of requirement from the GMC and Royal College website thoroughly at the beginning.

- Attending CESR Workshops could be helpful to understand the process. 
- Find a mentor, supervisor or buddy and also seek advice from someone who has gone through the process.

- Be familiar with all the paperwork and competencies right from the start. It will be helpful to chalk out the strategy for your training. - Be disciplined with paperwork and have a clear vision of your goals to achieve.

- Work on your goals by dividing them small, realistic and achievable milestones.

- Time is of the essence as you need to have all relevant paperwork within 5 years as per GMC guidance.

- This should not be considered as a short-cut to achieve CCT if you don't have adequate experience. This might take longer than formal training.

- Trainees can be really helpful colleagues. Ask for help when uncertain about how paperwork could be collated to evidence, knowledge and skills in clinical/non-clinical domains.

- When accepting a job at any trust, make sure they understand your training needs and agree with the level of support needed for CESR.

- Get your colleagues on board right from the beginning (i.e. CSL/line manager, College Tutor, Rota manager, module leads) and let them know your training needs. This will help to make a successful strategy. Communication with colleagues is essential \& it can determine your success.

-Work on your CV to make it more appealing for job applications.

- The onus is always on you to make arrangements for your training and progress. You need to have an absolute burning desire to progress.

- Lastly, I would like to say that most of the difficult accomplishments in the world have been achieved by people who kept on trying when there seemed to be no hope at all. So be resilient. There will be times when you feel low, be patient. If you are motivated, people will help you to achieve your goal.
Many CESR aspirant wonder if it is difficult to get a substantive consultant post if application is successful. There is no straightforward answer to this question. You are equivalent to any Post CCT UK trainee when your name is added to a Specialist register maintained by the GMC. There are many trusts employing CESR candidates, however, some trusts prefer a candidate from the UK training program. It also depends on the personal specification of the post as well as what you can offer to the hospital similar to any other job available in the market. Good personal attributes and relations within the department will help to secure a job as a consultant.

I hope my fellow CESR aspirants will agree with my views \& this advice could be useful to others who are thinking about this route.

"You're braver than you believe, and stronger than you seem, and smarterthan you think". -A.A.Milne

\section{Goodluck!}

\section{Useful links to read further and for guidance:}

1. https://www.gmc-uk.org/registration-and-licensing/join-theregister/registration-applications/specialist-applicationguides/specialist-registration-cesr-or-cegpr

2. https://www.gmc-uk.org/registration-and-licensing/join-theregister/registration-applications/specialty-specific-guidancefor-cesr-and-cegpr

3. https://www.gmc-uk.org/-/media/documents/sat---cesrcegpr-online-application---user-guide---dc11550_pdf76194730.pdf

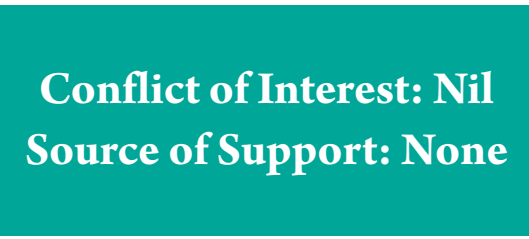

\section{How to Cite this Article}

Patki C | Road to CESR (Article 14)- Is it for me? | Journal of Anaesthesia and Critical Care Case Reports | May-August 2021; 7(2): 03-05. 